\title{
A plant Y chromosome-STS marker encoding a degenerate retrotransposon
}

\author{
Mari Obara $^{1, *}$, Sachihiro Matsunaga ${ }^{1, \#}$, Shunsuke Nakao ${ }^{2}$ and Shigeyuki Kawano ${ }^{1,2}$ \\ ${ }^{1}$ Department of Integrated Biosciences, Graduate School of Frontier Sciences, University of Tokyo, \\ Bldg. FSB-601, 5-1-5 Kashiwanoha, Kashiwa, Chiba 277-8562, Japan \\ ${ }^{2}$ Department of Biological Sciences, Graduate School of Sciences, University of Tokyo, \\ 7-3-1 Hongo, Tokyo 113-0033, Japan
}

(Received 30 April 2002, accepted 15 October 2002)

\begin{abstract}
The dioecious plant Silene latifolia has both X and Y sex chromosomes. Malespecific random amplified polymorphic DNA (RAPD) fragments were analyzed to identify Y-chromosome-linked sequences. One of the RAPD fragments, MS4, was converted into a more reliable and reproducible sequence-tagged site (STS) marker. A set of MS4 STS primers was used to amplify two genomic DNA fragments (MS4a and MS4b) from a male plant and one (MS4a) from a female plant, which indicates that MS4b is located on the Y chromosome. Sequence analysis revealed that MS4a encoded a gag protein of a Ty3-gypsy-like retrotransposon. A 147-bp region from the middle of MS4a was deleted in MS4b. The MS4b sequence was not detected in the most closely related dioecious species, $S$. dioica. This suggests that a retrotransposon with the MS4b sequence has degenerated recently on the $\mathrm{Y}$ chromosome.
\end{abstract}

\section{INTRODUCTION}

Approximately $6 \%$ of angiosperm species are dioecious, with separate production of staminate and pistillate flowers (Renner and Ricklefs, 1995). Heteromorphic sex chromosomes have been reported in several dioecious species (Matsunaga and Kawano, 2001, Negrutiu et al., 2001). The dioecious plant Silene latifolia has X and Y sex chromosomes, which are the largest and second largest chromosomes in the male genome, respectively (Matsunaga et al., 1994). When fluorescence in situ hybridization was performed with microdissected Y chromosome-DNA in the presence of competitor female genomic DNA, the signals on the $\mathrm{Y}$ chromosome were no stronger than those on the $\mathrm{X}$ chromosome and autosomes (Matsunaga et al., 1999a). This suggests that the $\mathrm{Y}$ chromosome of S. latifolia has not accumulated chromosome-specific sequences and has not become heterochromatic. An interstitial telomere-adjacent sequence is accumulated in the interstitial region of the Y chromosome, suggesting past telomere-mediated chromosome rearrangements during the evolution of the sex chromo-

\footnotetext{
Edited by Eiichi Ohtsubo

* Corresponding author and Present address: Department of Biotechnology, Graduate School of Engineering, Osaka University 2-1 Yamadaoka, Suita 565-0871, Osaka, Japan

E-mail: sachi@bio.eng.osaka-u.ac.jp

* Present address: Laboratory of Plant Breeding and Genetics, Graduate School of Agricultural and Life Sciences, University of Tokyo, 1-1-1 Yayoi, Bunkyo-ku, Tokyo 113-8657, Japan
}

somes of S. latifolia (Uchida et al., 2002). This primitive characteristic of the sex chromosomes provides empirical data on the initial evolution of sex chromosomes.

The function of the Y chromosome of S. latifolia was first revealed by analyzing mutant offspring with aberrant $\mathrm{Y}$ chromosomes from crosses that involved experimentally raised tetraploids (Westergaard, 1946). These analyses led to the hypothesis that the regulatory genes for female suppression, stamen promotion, and stamen maturation were located on the Y chromosome. Recently, this hypothesis has gained support from genetic and cytological analyses of hermaphroditic and asexual mutants that were generated by X-ray or $\gamma$-ray irradiation of pollen (Donnison et al., 1996; Farbos et al., 1999; Lardon et al., 1999). Male-specific amplified fragment length polymorphism (AFLP) markers were identified using X-rayinduced deletions of the $\mathrm{Y}$ chromosome (Lebel-Hardenack et al., 2002). Thus, three different loci of carpel suppression, early stamen development, and late stamen development have been located on the Y chromosome.

The random amplified polymorphic DNA (RAPD) method has provided a number of useful genetic markers in plants (Williams et al., 1990). In the case of dioecious plants, DNA fragments that were amplified only from the male genome were expected to be located on the Y chromosome (Mulcahy et al., 1992; Sakamoto et al., 1995). Several Y-chromosome-linked RAPD markers have been converted into more reliable sequence-tagged site (STS) markers (Zhang et al., 1998; Nakao et al., 2001). We report a novel STS marker that encodes a degenerate 
LTR retrotransposon on the Y chromosome.

The internal domain of the LTR retrotransposon encodes proteins that are homologous to retroviral protease, RNase $\mathrm{H}$, reverse transcriptase and integrase. LTR retrotransposons are divided into two types based on their protein arrangements: the Ty1-copia-like and Ty3gypsy-like retrotransposons, which were first reported in yeast (Clare and Farabaugh, 1985) and the fruit fly (Mount and Rubin, 1985), respectively. Both retrotransposon types are found in almost all plants (Grandbanstien, 1992). In this paper, we report a degenerate Ty3gypsy retrotransposon sequence that is linked to the $\mathrm{Y}$ chromosome.

\section{MATERIALS AND METHODS}

Plant materials and DNA isolation. Seeds of Silene latifolia Poir. ssp. alba (P. Mill.) Greuter and Burdet were obtained from an inbred line in our laboratory. Seeds of S. dioica (L.) Clairville were collected from an herb garden near Lake Kawaguchi (Yamanashi, Japan). Plants were grown in flowerpots at $22^{\circ} \mathrm{C}$ in a temperature-regulated chamber. Genomic DNA was isolated from young male and female leaves using a Nucleon PhytoPure Kit (Amersham Pharmacia Biotech, Buckinghamshire, UK).

Isolation of RAPD and sequence-tagged site (STS) PCR analysis. The PCR reaction mixture contained $200 \mu \mathrm{M}$ of each dNTP, $0.4 \mu \mathrm{M}$ each of two arbitrary 10 mer primers (Wako Chemicals, Japan), $50 \mathrm{ng}$ of genomic DNA as the template, $1 \mathrm{U}$ of Takara ExTaq DNA polymerase (Takara Shuzo, Otsu, Japan), and $1 \times$ ExTaq buffer (Takara Shuzo), in a total volume of $50 \mu \mathrm{l}$. After an initial denaturation step at $94^{\circ} \mathrm{C}$ for $3 \mathrm{~min}$, the samples were subjected to 35 cycles of $94^{\circ} \mathrm{C}$ for $1 \mathrm{~min}, 35^{\circ} \mathrm{C}$ for $1 \mathrm{~min}$, and $72^{\circ} \mathrm{C}$ for $2 \mathrm{~min}$. An aliquot of the PCR reaction $(5 \mu \mathrm{l})$ was electrophoresed on a $4 \%$ polyacrylamide gel and stained with SYBR Green I (Molecular Probes, OR, USA).

Two 18-mer STS primers, MS4-R (5'-GGACACGATGACACCAAC-3') and MS4-F (5'-AGGCGTTGACCAGTTCAG-3'), were used for the STS-PCR analysis. The PCR reaction mixture contained $200 \mu \mathrm{M}$ of each dNTP, $0.4 \mu \mathrm{M}$ of each primer, $50 \mathrm{ng}$ of genomic DNA as the template, 1 U of Takara ExTaq DNA polymerase (Takara Shuzo), and $1 \times$ ExTaq buffer (Takara Shuzo), in a total volume of $50 \mu \mathrm{l}$. After an initial denaturation step at $94^{\circ} \mathrm{C}$ for $3 \mathrm{~min}$, the samples were amplified for 35 cycles of $94^{\circ} \mathrm{C}$ for $1 \mathrm{~min}, 1 \mathrm{~min}$ at $58^{\circ} \mathrm{C}$ for S. latifolia or $54^{\circ} \mathrm{C}$ for $S$. dioica, and $72^{\circ} \mathrm{C}$ for $1 \mathrm{~min}$ (for S. latifolia). An aliquot of the PCR reaction $(5 \mu \mathrm{l})$ was electrophoresed on a $2 \%$ agarose gel.

Southern hybridization analysis. Genomic DNA samples from $S$. latifolia male and female tissues were digested with HindIII. Samples $(4 \mu \mathrm{g})$ were electrophoresed on $1.5 \%$ agarose gels, transferred to a Biodyne B nylon membrane (Pall Biosupport Division, NY, USA) using a G capillary blotter (TIETEC, Nagoya, Japan), and fixed with a Spectrolinker XL-1500 UV cross-linker (Spectronics). Probe preparation, hybridization and detection were performed using an ECL Direct Nucleic Acid Labeling and Detection System (Amersham). When MS4a and MS4b were used as probes, the primary washes consisted of $2 \times \mathrm{SSC}$ at $42^{\circ} \mathrm{C}$ and $0.1 \times \mathrm{SSC}$ at $42^{\circ} \mathrm{C}$, respectively. The hybridization membrane was exposed to X-OMAT AR film (Kodak, USA) at room temperature. The MS4a and MS4b hybridization membranes were exposed for $45 \mathrm{~min}$ and $16 \mathrm{~h}$, respectively.

Sequence analyses. The nucleotide sequences were determined by the dideoxy chain termination method using a 373S DNA Sequence System and the THERMO Sequenase II Dye Terminator Cycle Sequencing Kit (Amersham) or the Dynamic ET Terminator Cycle Sequencing Kit (Amersham Pharmacia Biotech). The BLASTN and BLASTX programs (Altschul et al., 1990) were used for similarity searches of the DDBJ/EMBL/ GenBank databases. The sequences were analyzed using the DNA Space software (Hitachi Software Engineering Co., Ltd., Hitachi, Japan).

Accession numbers. The DDBJ/EMBL/GenBank accession numbers of MS4a and MS4b are AB072492 and $\mathrm{AB} 072494$, respectively.

\section{RESULTS AND DISCUSSION}

We performed RAPD analysis using male and female genomic DNA samples in order to isolate male-specific DNA sequences from the $\mathrm{Y}$ chromosome. Using a total of 94 combinations of arbitrary 10-mer primers, 29 malespecific RAPDs were obtained. The male-specific RAPDs ranged in size from approximately 0.1 to $1.2 \mathrm{~kb}$, and three of these sequences were converted successfully to STS markers for the $\mathrm{Y}$ chromosome. In this paper, we report the detailed analysis of one of these STS markers. Amplification with the CT63 (5'-AGGCGTTGAC-3') and CT65 (5'-GGACACGATG-3') primers revealed that a male-specific RAPD, which we named MS4, was ubiquitous in males and absent in females (Fig. 1a). The RAPD fragment was cloned and sequenced, and based on the sequencing results, we designed the 18-mer primers MS4-R (5'-GGACACGATGACACCAAC-3') and MS4-F (5'AGGCGTTGACCAGTTCAG-3'). As shown in Fig. 1a, the MS4 primer set amplified two types of fragment from the male and female genomic DNAs of four individuals; we named the longer fragment MS4a, and the shorter fragment MS4b. MS4a was amplified from both male and female genomic DNAs, whereas MS4b was amplified 
a

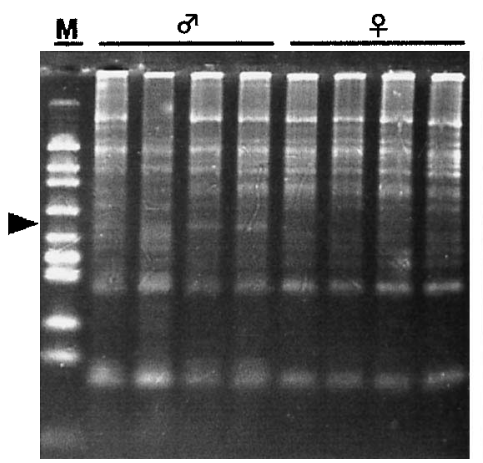

b

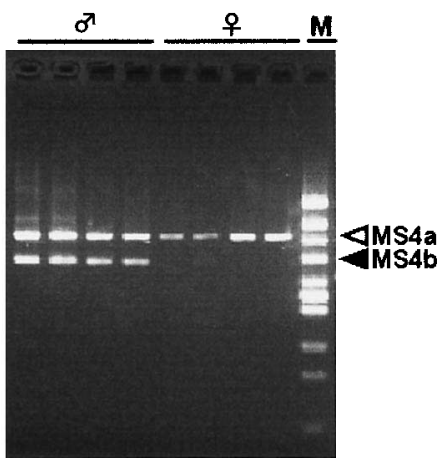

Fig. 1. RAPD and STS-PCR profiles of four male and four female individuals.

PCR amplification was performed using genomic DNA from four male ( $\delta$ ) and four female ( 9 ) S. latifolia plants. (a) RAPD profile of S. latifolia genomic DNA. Fragments were amplified with the CT63 and CT65 primers, separated on a 4\% polyacrylamide gel, and stained with SYBR Green I. The solid arrowhead indicates a male-specific fragment, MS4. (b) Fragments were amplified with the MS4 primer set, separated on a 3\% agarose gel, and stained with ethidium bromide. The open and solid arrowheads indicate the 596-bp common fragment (MS4a) and the 443-bp male-specific fragment (MS4b), respectively. Lane M contains HincII-digested $\phi X 174$ DNA as the molecular weight marker.

only from male genomic DNA. The PCR results obtained with the MS4 primer set were reproduced with the DNA from ten male and female individuals from five different strains (data not shown). This suggests that MS4a is localized on the autosome, the $\mathrm{X}$ chromosome, or pseudoautosomal regions of the sex chromosomes, while MS4b is located only on the Y chromosome.

In order to analyze the relationship between MS4a and MS4b, the two fragments were cloned and sequenced. The MS4a fragment contained a complete open reading frame of 198 amino acids, and the nucleotide sequences of MS4a were perfectly identical in males and females. Database searching revealed that MS4a shared homology with several gag proteins of Ty3-gypsy-like retrotransposons in Arabidopsis thaliana and Oryza sativa (Fig. 2). Most of the gag proteins in O. sativa have significant homology to the Ty3-gypsy-like retrotransposon, RIRE2 (Ohtsubo et al., 1999). These results indicate that MS4a encodes a gag protein in a Ty3-gypsy-like retrotransposon. Retrotransposons are commonly found in plant species (Kumekawa et al., 1999; Noma et al., 1999). Southern blots and fluorescence in situ hybridization analysis revealed that Ty3-gypsy-like retrotransposons were distributed throughout the genome and did

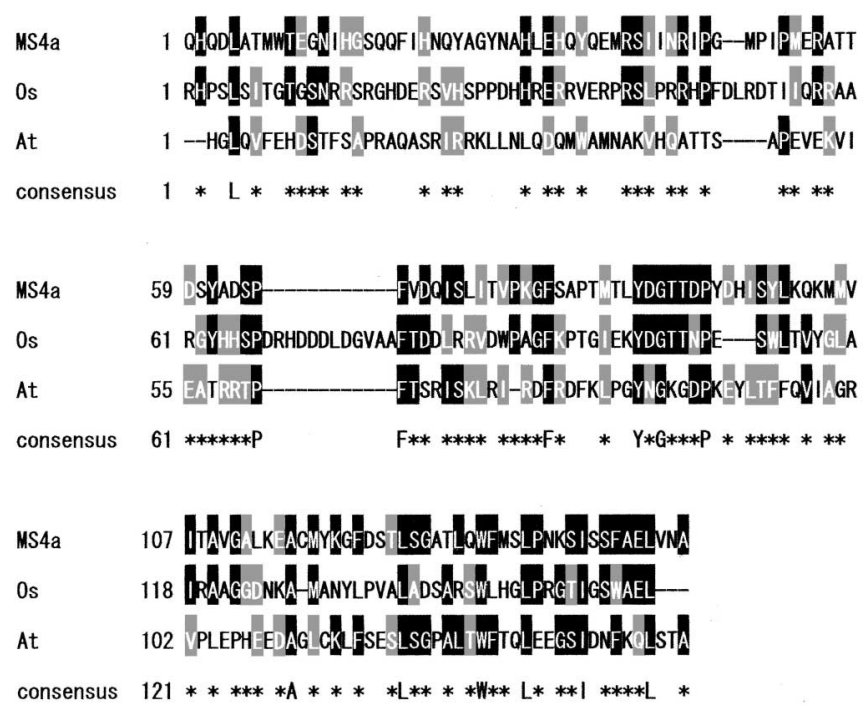

Fig. 2. Comparison of MS4a amino acid sequences. The solid and shaded boxes indicate similar and identical amino acids, respectively. Capital letters and asterisks indicate perfect and imperfect consensus sequences, respectively. The 'At' designation signifies a hypothetical gag-pol protein of Arabidopsis thaliana (accession no. AC006085), and 'Os' represents the gag-pol protein that is encoded by a retrotransposon in Oryza sativa (accession no. AF111709). 
not accumulate preferentially on the Y chromosome of $S$. latifolia (Matsunaga et al., 2002). In order to determine the genomic organization of MS4a, Southern hybridization analysis was performed using HindIII-digested genomic DNA from four males and four females. MS4a hybridized to several fragments, and gave identical hybridization patterns in the male and female genomes. This result suggests that MS4a is one of the repetitive sequences that are dispersed over the male and female genomes (Fig. 3a). The genomic distribution of MS4a (Fig. 3a) is consistent with previous analyses of Ty3-

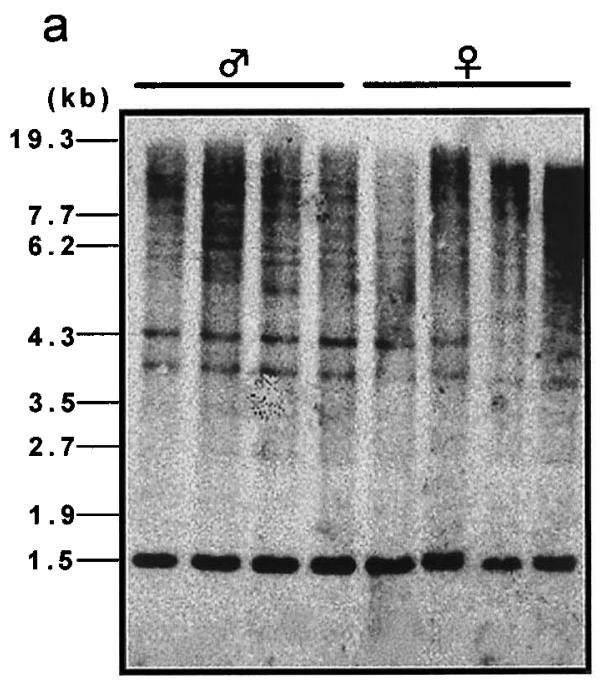

\section{b}

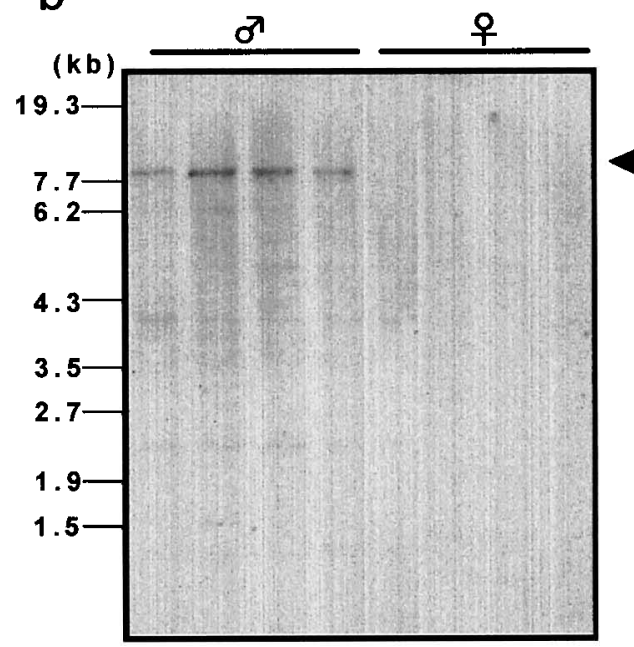

Fig. 3. Genomic distribution of MS4a and MS4b in male and female genomes. Southern hybridization was performed using HindIII-digested genomic DNA from four male ( $\hat{\delta})$ and four female ( $q$ ) plants. HindIII-digested genomic DNAs were separated on a $1.5 \%$ agarose gel. Labeling, hybridization and detection were performed using the ECL Direct System (Amersham). (a) The blotted membrane was hybridized with the MS4a probe. (b) The blotted membrane was hybridized with the MS4b probe at high stringency. The arrowhead indicates an MS4b-hybridizing fragment. gypsy-like retrotransposons (Matsunaga et al., 2002).

On the other hand, comparison of the nucleotide sequences of MS4a and MS4b suggests that MS4b originated mainly through deletion of the middle region of MS4a, as shown in Fig. 5. A 147-bp central region of MS4a is deleted in MS4b. Moreover, the putative MS4b ORF has three stop codons, whereas MS4a encompasses a retrotransposon that lacks stop codons. This indicates that the retrotransposon with the MS4b sequence is inactive due to degeneration. When the hybridization experiment was performed at high stringency, MS4b hybridized specifically to an approximately 8 -kb fragment in males (Fig. 3b). This finding supports our contention that MS4b is located on the $\mathrm{Y}$ chromosome. In fact, a set of these STS primers, MS4-R and MS4-F, is very useful for genetical determination of sex for two reasons as follows. One reason is that MS4b is a stable STS marker for males because the gypsy-type retrotransposon including MS4b is inactive on the Y chromosome. The other is that false negatives are eliminated from PCR samples because this primer set can simultaneously amplify a non-sex linked fragment MS4a.

We wished to confirm that MS4a and MS4b were located on the $\mathrm{Y}$ chromosome of a closely related dioecious species, S. dioica. The genomic DNA of $S$. dioica was subjected to PCR with the MS4F and MS4R primers. As shown in Figure 4, the primers amplified MS4a sequences in both male and female $S$. dioica individuals. The MS4a fragments in S. dioica were cloned and sequenced, to reveal a nucleotide sequence identity of $99.7 \%$ between male and female individuals. The discrepancy in sequence identity is due to polymorphisms, since $S$. dioica was derived from a wild population rather than an experimental line. MS4a was $95.7 \%$ identical in S. latifolia and S. dioica (Fig. 5). In contrast, MS4b could not be detected in male $S$. dioica. This pattern was reproduc-

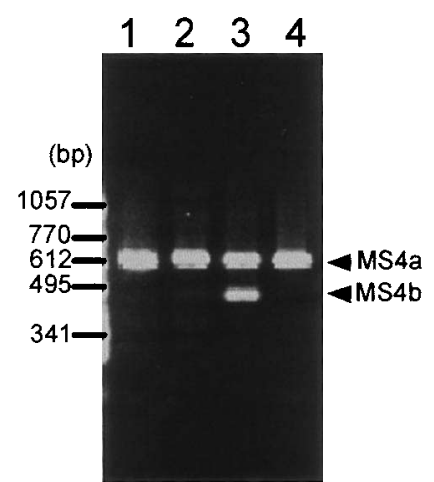

Fig. 4. Analysis of MS4 homologues in S. dioica. MS4 homologues were amplified using the MS4F and MS4R primers. PCR products were separated on a $3 \%$ agarose gel and stained with ethidium bromide. Lanes $1,2,3$ and 4 show the PCR products from the genomic DNA of male $S$. dioica, female $S$. dioica, male $S$. latifolia and female $S$. latifolia plants, respectively. 


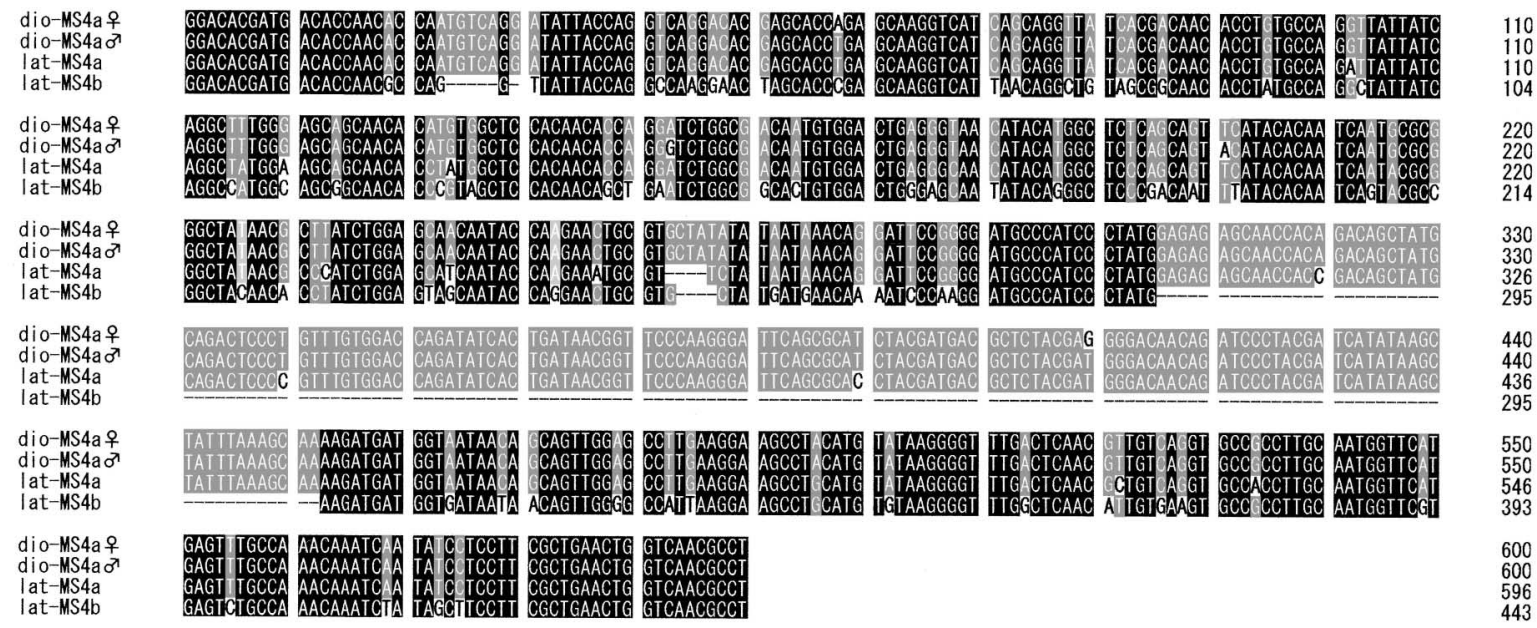

Fig. 5. Alignment of the MS4 genes of S. latifolia and S. dioica.

Dio-MS4a o and dio-MS4a of indicate the MS4a nucleotide sequences in male and female S. dioica individuals, respectively. LatMS4a and lat-MS4b represent the MS4b nucleotide sequences in male and female S. latifolia individuals, respectively. The solid and shaded boxes represent fully and partially identical nucleotides, respectively.

ible in two other strains of $S$. dioica (data not shown). These results suggest that at least regions of MS4b are not conserved on the Y chromosomes of S. latifolia and $S$. dioica. It is possible that the MS4b sequence emerged in S. latifolia after divergence from $S$. dioica.

It seems likely that the retrotransposon with MS4b lost function after moving to the Y chromosome of $S$. latifolia. A male reproductive organ-specific gene, MROS3, is located on the $\mathrm{X}$ chromosome (Matsunaga et al., 1996; Guttman and Charlesworth, 1998; Matsunaga et al., 1999b; Kejnovsky et al., 2001). A paralogous sequence on the $\mathrm{Y}$ chromosome, MROS3Y, is highly homologous to the 3 '-region of the expressed MROS3 sequence (Guttman and Charlesworth, 1998). MROS3Y appears to have become non-functional following degeneration of the $\mathrm{Y}$ chromosome. The analysis of MS4b also offers evidence of Y chromosome degeneration in $S$. latifolia. More refined analyses of MS4b will provide interesting data regarding the process of differentiation in plant sex chromosomes.

This work was supported by Grants-in-aid for Scientific Research on Priority Areas (C) Genome Biology from the Ministry of Education, Culture, Sports, Science and Technology of Japan and Research for the Future from the Japan Society for the Promotion of Science.

\section{REFERENCES}

Altschul, S. F., Gish, W., Miller, W., Myers, E. W., and Lipman, D. J. (1990) Basic local alignment search tool. J. Mol. Biol. 215, 403-410.

Clare, J., and Farabaugh, P. (1985) Nucleotide sequence of a yeast Ty element: evidence for an unusual mechanism of gene expression. Proc. Natl. Acad. Sci. USA 82, 28292833.

Donnison, I. S., Siroky, J., Vyskot, B., Saedler, H., and Grant, S.
R. (1996) Isolation of Y chromosome-specific sequences from Silene latifolia and mapping of male sex-determining genes using representational difference analysis. Genetics 144, 1893-1901.

Farbos, I., Veuskens, J., Vyskot, B., Oliveira M., Hinnisdaels, S., Aghmir, A., Mouras, A., and Negrutiu, I. (1999) Sexual dimorphism in white campion: deletion on the $\mathrm{Y}$ chromosome results in a flora asexual phenotype, Genetics 151, $1187-1196$.

Grandbastien, M-A. (1992) Retroelements in higher plants. Trends Genet. 8, 103-108.

Guttman, D. S., and Charlesworth, D. (1998) An X-linked gene has a degenerate Y-linked homologue in the dioecious plant Silene latifolia. Nature 393, 263-266.

Kejnovsky, E., Vrana, J., Matsunaga, S., Soucek, P., Siroky, J., Dolezel, J., and Vyskot, B. (2001) Localization of male-specifically expressed MROS genes of Silene latifolia by PCR on flow-sorted X chromosomes and autosomes. Genetics 158, 1269-1277.

Kumekawa, N., Ohtsubo, E., and Ohtsubo, H. (1999) Identification and phylogenetic analysis of gypsy-type retrotransposons in the plant kingdom. Genes Genet. Syst. 74, 299307.

Lardon, A., Georgiev, S., Aghmir, A., Le Merrer G., and Negrutiu, I. (1999) Sexual Dimorphism in white campion: complex control of carpel number is revealed by $\mathrm{Y}$ chromosome deletions. Genetics 151, 1173-1185.

Level-Hardenack, S., Hauser, E., Law, T. F., Schmid, J., and Grant, S. R. (2002) Mapping of sex determination loci on the white campion (Silene latifolia) Y chromosome using amplified fragment length polymorphism. Genetics 160, 717725 .

Matsunaga, S., Hizume, M., Kawano, S., and Kuroiwa, T. (1994) Cytological analyses in Melandrium album: genome size, chromosome size and fluorescence in situ hybridization. Cytologia 59, 135-141.

Matsunaga, S., Kawano, S., Takano, H., Uchida, H., Sakai, A., and Kuroiwa, T. (1996) Isolation and development expression of male reproductive organ-specific genes in a dioecious campion, Melandrium album (Silene latifolia). Plant J. 10, 679-639. 
Matsunaga, S., Kawano, S., Michimoto, T., Higashiyama, T., Nakao, S., Sakai, A., and Kuroiwa, T. (1999a) Semi-automatic laser beam microdissection of the $\mathrm{Y}$ chromosome and analysis of Y chromosome DNA in a dioecious plant, Silene latifolia. Plant Cell Physiol. 40, 60-68.

Matsunaga, S., Schueze, K., Donnison I., Grant S. R., Kuroiwa T., and Kawano S. (1999b) Single pollen typing combined with laser-mediated manipulation. Plant J. 20, 371-378.

Matsunaga, S., and Kawano S. (2001) Sex determination by sex chromosomes in dioecious plants. Plant Biology 3, 1-8.

Matsunaga, S., Yagisawa, F., Yamamoto, M., Uchida, W., Nakao, S., and Kawano S. (2002) LTR retrotransposons in the dioecious plant Silene latifolia. Genome 45, 745-751.

Mulcahy, D. L., Weeden, N. F., Kesselei, R., and Carroll, S. B. (1992) DNA probes for the Y chromosome of Silene latifolia, a dioecious angiosperm. Sex. Plant Reprod. 5, 86-88.

Mount, S. M., and Rubin, G.M. (1985) Complete nucleotide sequence of the Drosophila transposable element copia: homology between copia and retroviral proteins. Mol. Cell. Biol. 5, 1630-1638.

Nakao, S., Matsunaga, S., Sakai, A., Kuroiwa, T., and Kawano, S. (2002) RAPD isolation of a Y chromosome-specific ORF in a dioecious plant, Silene latifolia. Genome 45, 413-420.

Negrutiu, I., Vyskot, B., Barbacar, N., Georgiev, S., and Moneger, F. (2001) Dioecious plants. A key to the early events of sex chromosome evolution. Plant Physiol. 127, 1418-1424.

Noma, K., Ohtsubo, E., and Ohtsubo, H. (1999) Non-LTR ret- rotransposons (LINEs) as ubiquitous components of plant genomes. Mol. Gen. Genet. 261, 71-79.

Ohtsubo, H., Kumekawa, N., and Ohtsubo, E. (1999) RIRE2, a novel gypsy-type retrotransposon from rice. Genes Genet. Syst. 74, 83-91.

Renner, S. S., and Ricklefs, R. E. (1995) Dioecy and its correlates in the flowering plants. Am. J. Bot. 82, 596-606.

Sakamoto, K., Shimomura, K., Komeda, Y., Kamada, H. and Satoh, S. (1995) A male-associated DNA sequence in a dioecious plant, Cannabis sativa L. Plant Cell Physiol. 36, 1549-1554.

Uchida W., Matsunaga S., Sugiyama R., Shibata F., Kazama Y., Miyazawa Y., Hizume M., Kawano S. (2002) Distribution of interstitial telomere-like repeats and their adjacent sequences in a dioecious plant, Silene latifolia. Chromosoma, 111, 313-320.

Westergaard, M. (1946) Aberrant Y chromosomes and sex expression in Melandrium album. Hereditas 32, 419-431.

Williams, J. G. K., Kubelik, A. R., Livak, K. J., Rafalski, J. A., and Tingey, S. V. (1990) DNA polymorphisms amplified by arbitrary primers are useful as genetic markers. Nucleic Acids Res. 18, 6531-6535.

Zhang, Y. H., Di Stilio, V. S., Rehman, F., Avery A., Mulcahy D., and Kesseli R. (1998) Y chromosome-specific markers and the evolution of dioecy in the genus Silene. Genome 41, 141-147. 